\title{
A preoperative nomogram for sepsis in percutaneous nephrolithotomy treating solitary, unilateral, and proximal ureteral stones
}

\author{
Yang Xun ${ }^{1}$, Yuanyuan Yang ${ }^{1}$, Xiao Yu ${ }^{1}$, Cong Li ${ }^{1}$, Junlin Lu ${ }^{\text {Corresp., } 1}{ }^{\text {, Shaogang Wang }}{ }^{\text {Corresp. } 1}$ \\ ${ }^{1}$ Department of Urology, Tongji Hospital, Tongji Medical College, Huazhong University of Science and Technology, Wuhan, China \\ Corresponding Authors: Junlin Lu, Shaogang Wang \\ Email address: lujunlintjm@126.com, sgwangtjm@163.com
}

BACKGROUND: Postoperative sepsis is a lethal complication for percutaneous nephrolithotomy (PCNL). An early predictive model combined local and systemic conditions is urgently needed to predict infectious events. We aim to determine the preoperative predictors of sepsis after PCNL in patients with unilateral, solitary, and proximal ureteral stones.

METHODS: A total of 745 patients who underwent PCNL between January 2012 and December 2018 were retrospectively enrolled. Sepsis was defined based on the International Sepsis Definitions in 2001, and the preoperative factors were compared between the non-sepsis and sepsis groups. Univariable analysis and multivariable logistic regression analysis were conducted to determine the predictors for sepsis after PCNL. A nomogram was generated using the predictors.

RESULTS: In this study, 35 patients (4.7\%) developed sepsis after PCNL. Univariate analysis showed that post-PCNL sepsis was associated with the female, lower albumin, higher globulin, lower albumin globulin ratio (AGR <1.5), preoperative fever, leukocytosis (WBC $\geq 10,000$ cells/ $\mu \mathrm{L}$ ), positive urine culture, leukocyturia ( $\geq 50$ cells $/ \mu \mathrm{L}$ ) and positive urine nitrite. Multivariate logistic regression analysis suggested that $A G R<1.5$ (odds ratio $[O R]=5.068,95 \%$ confidence interval $[\mathrm{Cl}]=1.135-22.624, \mathrm{P}=0.033$ ), positive urine culture $(\mathrm{OR}=3.243,95 \% \mathrm{Cl}=1.162-9.047, \mathrm{P}=0.025)$, leukocytosis $(\mathrm{OR}=3.706,95 \% \mathrm{Cl}=1.444-9.512$, $\mathrm{P}=0.006)$, and female $(\mathrm{OR}=2.529,95 \% \mathrm{Cl}=1.127-5.672, \mathrm{P}=0.024)$ were independent risk factors for sepsis. A nomogram was generated and displayed favorable fitting (Hosmer-Lemeshow test $P=0.797$ ), discrimination (area under receiver operating characteristic curve was 0.807 ), and clinical usefulness by decision curve analysis.

CONCLUSIONS: Patients with certain preoperative characteristics, such as female, lower AGR, positive urine culture, and leukocytosis, who undergo PCNL may have a higher risk of developing sepsis. A cautious preoperative evaluation and optimized treatment strategy should be considered in these patients to minimize infectious complications. 


\section{Title: A preoperative nomogram for sepsis in}

2 percutaneous nephrolithotomy treating solitary,

3 unilateral, and proximal ureteral stones

4

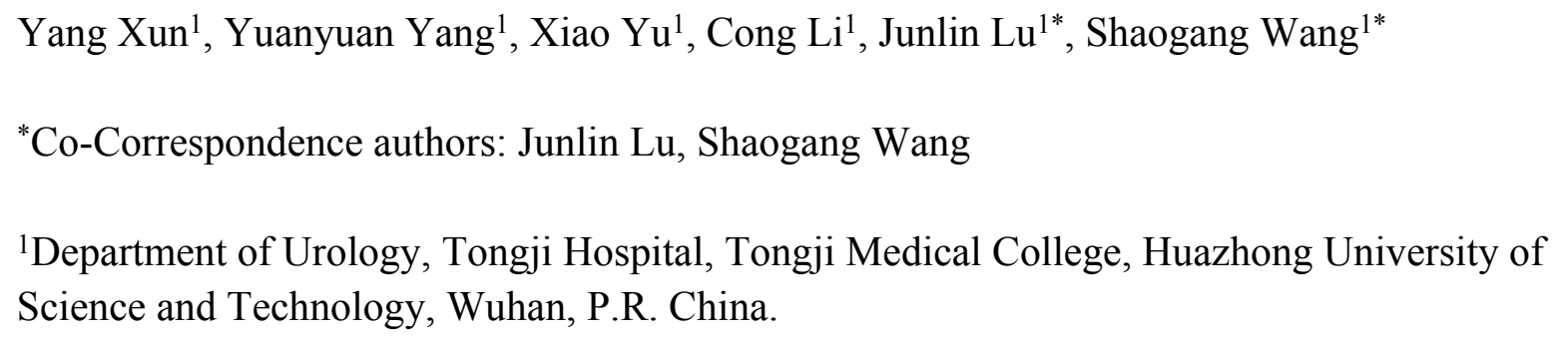

Correspondence 1: Junlin Lu, Department of Urology, Tongji Hospital, Tongji Medical College, Huazhong University of Science and Technology, Wuhan, P.R. China. E-mail: lujunlintjm@126.com Telephone numbers: +86-27-83693756

Correspondence 2: Shaogang Wang, Department of Urology, Tongji Hospital, Tongji Medical College, Huazhong University of Science and Technology, Wuhan, P.R. China. E-mail: sgwangtjm@163.com Telephone numbers: Tel.+86-027-83663460.

\section{Abstract}

BACKGROUND: Postoperative sepsis is a lethal complication for percutaneous nephrolithotomy (PCNL). An early predictive model combined local and systemic conditions is urgently needed to predict infectious events. We aim to determine the preoperative predictors of sepsis after PCNL in patients with unilateral, solitary, and proximal ureteral stones.

METHODS: A total of 745 patients who underwent PCNL between January 2012 and December 2018 were retrospectively enrolled. Sepsis was defined based on the International Sepsis Definitions in 2001, and the preoperative factors were compared between the non-sepsis and sepsis groups. Univariable analysis and multivariable logistic regression analysis were conducted to determine the predictors for sepsis after PCNL. A nomogram was generated using the predictors.

RESULTS: In this study, 35 patients (4.7\%) developed sepsis after PCNL. Univariate analysis showed that post-PCNL sepsis was associated with the female, lower albumin, higher globulin, lower albumin globulin ratio (AGR $<1.5$ ), preoperative fever, leukocytosis ( $\mathrm{WBC} \geqslant 10,000$ cells/ $\mu \mathrm{L}$ ), positive urine culture, leukocyturia ( $\geqslant 50$ cells/ $\mu \mathrm{L}$ ) and positive urine nitrite. Multivariate logistic regression analysis suggested that AGR $<1.5$ (odds ratio $[\mathrm{OR}]=5.068,95 \%$ confidence interval $[\mathrm{CI}]=1.135-22.624, \mathrm{P}=0.033)$, positive urine culture $(\mathrm{OR}=3.243,95 \%$ $\mathrm{CI}=1.162-9.047, \mathrm{P}=0.025)$, leukocytosis $(\mathrm{OR}=3.706,95 \% \mathrm{CI}=1.444-9.512, \mathrm{P}=0.006)$, and 
40 female $(\mathrm{OR}=2.529,95 \% \mathrm{CI}=1.127-5.672, \mathrm{P}=0.024)$ were independent risk factors for sepsis. A

41 nomogram was generated and displayed favorable fitting (Hosmer-Lemeshow test $\mathrm{P}=0.797$ ),

42 discrimination (area under receiver operating characteristic curve was 0.807), and clinical

43 usefulness by decision curve analysis.

44 CONCLUSIONS: Patients with certain preoperative characteristics, such as female, lower AGR, positive urine culture, and leukocytosis, who undergo PCNL may have a higher risk of

47

48

49

50

51

52

53

54

55

56

57

58

59

60

61

62

63

64

65

66

67

68

69

70

71

72

73

74

75

76

77

78

\section{1}

6
developing sepsis. A cautious preoperative evaluation and optimized treatment strategy should be considered in these patients to minimize infectious complications.

\section{Introduction}

Urolithiasis is a common urologic disease with a prevalence rate of $5.8 \%$ in the general population, and the recurrence rate has been shown to reach $60 \%$ within 10 years ${ }^{1,2}$. The treatment options for stone disease range from open surgery to endoscopic surgery, such as percutaneous nephrolithotomy (PCNL) and ureteroscopy (URS) ${ }^{3}$. Generally, PCNL results in higher stone-free rate than URS, at the expense of elevated complication rate and prolonged hospital stays, without difference in secondary interventions ${ }^{4,5}$. However, infection remains a main complication that can cause longer length of stay and even lethal sepsis shock ${ }^{6}$. Therefore, establishing a predictive model, especially in the early stages of hospitalization, is imperative to help develop prevention strategies.

The risk factors for sepsis after PCNL were systematically summarized ${ }^{6}$. The preoperative factors comprised the following: positive urine culture, female sex, nephrostomy, urinary diversion, stone size, hydronephrosis, diabetes, and complicated stone. Among these, the local urological condition is considered to be a critical factor related to infectious complications. Additionally, new factors for infection have emerged in recent years. These factors, such as Creactive protein, albumin, and procalcitonin, show strong predictive value by reflecting the systematic condition of the patient ${ }^{7,8}$.

Currently, a preoperative model, integrating local and systematic conditions, is scarce to evaluate the probability of sepsis. The purpose of this study was to analyze post-PCNL sepsis in solitary, unilateral, and proximal ureteral stone patients. Furthermore, the results of this study were used to develop a preoperative risk factor nomogram that may help urologists identify patients who are more likely to develop sepsis.

als \& Methods

The study was conducted under the approval of the Ethics Committee of Tongji Medical College, Huazhong University of Science and Technology (2019S1035). We retrospectively enrolled 745 patients who underwent PCNL from January 2012 to December 2018. The inclusion criteria were the following: (1) PCNL was performed to treat unilateral, solitary, and 
79 proximal ureteral stones; and (2) age $\geqslant 18$ years. Stones size bigger than $10 \mathrm{~mm}$. The exclusion

80 criteria were anatomical renal abnormalities (horseshoe kidney, solitary kidney, transplant

81 kidney, and kidney duplication). Patients imaging, including abdominal computed tomography

$82 \mathrm{CT}$, confirmed the presence and size of proximal ureteral stones (above the fourth lumbar spine

83 or in the ureteropelvic junction). Types of anesthesia, preoperative antibiotics, surgeons' years'

84 experience, PCNL operation details were identified and recorded. Postoperative patients' data

85 were revised, collected, and recorded.

86 Patients information were collected from our hospital's database, including age, sex, body

87 mass index (BMI), comorbidities (diabetes, hypertension, and coronary heart disease), stone size

88 and laterality, prior indwelling stent, blood tests (cholesterol, creatinine, albumin, and globulin

89 levels and white blood cell count), fever (defined as body temperature $>38^{\circ} \mathrm{C}$ ), urine tests (white

90 blood cell and nitrite), urine culture, and ASA score.

91 Sepsis was defined according to the 2001 International Sepsis Definitions Conference;

92 occurrence of an infection and a minimum of two of the following within 48 hours of surgery:

93 (1) heart rate $>90 /$ minute, (2) body temperature $>38^{\circ} \mathrm{C}$, (3) leukocyte count $<4,000$ cells $/ \mu \mathrm{L}$ or

$94>12,000$ cells $/ \mu \mathrm{L}$, and (4) respiratory rate $>20 /$ minute 9.

95 All statistical analyses were performed using SPSS 24.0 and R software 3.6.2. The cut-off

96 value was determined by the Youden Index. The student t-test was used to detect differences

97 between continuous variables with a normal distribution. The chi-square test or Fisher's exact

98 test was used to compare categorical variables. The multivariable logistic regression method was

99 used to determine independent risk factors for sepsis. Then predictive nomogram was generated

100 based on converting the regression coefficient to a 0 - to 100-point scale proportionally. The

101 predictive performance of the model was measured by validation, discrimination and decision

102 analysis $^{10}$. A calibration curve was generated with 1000 bootstrap samples to reduce the overfit

103 bias. Hosmer-Lemeshow (HL) test implied good calibration when the test is insignificant. The

104 discriminative performance was assessed by area under the receiver operating characteristic

105 (ROC) curve. The clinical usefulness of the nomogram is evaluated by decision curve analysis

106 (DCA) by assessing net benefits at different threshold probabilities. P-value $<0.05$ was

107 considered significant.

108

109

110

\section{Results}

112 We identified 745 patients who underwent PCNL for a solitary, unilateral, and proximal

113 ureteral stone, and 35 patients developed sepsis. The patients' demographic details and

114 univariable analysis results of risk factors are shown in Table 1. No patients were concomitant

115 with paraplegia. The characteristics of patients who developed sepsis included female sex, lower

116 albumin, higher globulin, lower $\operatorname{AGR}(<1.5)$, preoperative fever, leukocytosis $(\mathrm{WBC} \geqslant 10,000$

117 cells/ $\mu \mathrm{L}$ ), positive urine culture, leukocyturia ( $\geqslant 50$ cells/ $\mu \mathrm{L}$ ), and positive urine nitrite. 
118 Notably, stone size and operation time were comparable between the two groups. The stone

119

120

121

122

123

124

125

126

127

128

129

130

131

132

133

134

135

136

137

138

139

140

141

142

143

144

145

146

147

148

149

150

151

152

153

154

155

156

157

shape is rotundity or extension.

In the multivariate analysis, four factors were identified as independent factors for sepsis: $\mathrm{AGR}<1.5$ (odds ratio $[\mathrm{OR}]=5.068,95 \%$ confidence interval $[\mathrm{CI}]=1.135-22.624, \mathrm{P}=0.033$ ), positive urine culture $(\mathrm{OR}=3.243,95 \% \mathrm{CI}=1.162-9.047, \mathrm{P}=0.025)$, leukocytosis $(\mathrm{OR}=3.706$, $95 \% \mathrm{CI}=1.444-9.512, \mathrm{P}=0.006)$, and female sex $(\mathrm{OR}=2.529,95 \% \mathrm{CI}=1.127-5.672, \mathrm{P}=0.024)$ (Table 2). Furthermore, we categorized AGR into 3 groups: $\geqslant 1.5,1.0-1.5$, and $<1.0$. Figure 1 shows an increasing sepsis rate with increasing levels: $0.78 \%(2 / 255), 5.93 \%(25 / 421)$, and $11.59 \%(8 / 69)$, respectively.

Based on the multivariable analysis, a nomogram prediction model was established to calculate the cumulative probability of sepsis after PCNL (Figure 2). The total points were obtained by adding the points assigned to the four factors. The corresponding sepsis rate is indicated by the total points axis. The calibration curve showed good fitting of the model with no statistical significance $(\mathrm{P}=0.797)$ through the Hosmer-Lemeshow test (Figure 3-A). The ROC curve illustrated favorable discrimination with an area under the curve of 0.807 (Figure 3-B). The DCA of the model showed a threshold probability of 10-90\% (Figure 4), in which the model had the ability to identify stone patients who might develop sepsis after PCNL superior to the "treat-all-patients" or "treat-none" schemes.

\section{Discussion}

Compared with other minimally invasive lithotripsy techniques, PCNL is generally considered to be a safe technique that offers the highest stone-free rates ${ }^{11,12}$. However, complications still occur following this percutaneous procedure. With the advancements in technology in recent years, the safety of PCNL has been effectively improved. However, infection is still one of the main complications. The sepsis rate reached 4.7\% (35/745) in our research, which was consistent with previous studies $(0.9 \%-5.9 \%)^{13,14}$. Once the infection further deteriorates without timely recognition and intervention, it may lead to life-threatening complications, like shock and organ dysfunction. Therefore, the early determination of factors associated with sepsis complications is critical to avoid serious postoperative events. In this study, we developed a nomogram utilizing the four preoperative characteristics from multivariate analysis: sex, AGR, urine culture, and blood WBC. This preoperative predictive model combined both local and systematic conditions to evaluate the probability of sepsis.

Interestingly, our study revealed that $\mathrm{AGR}(<1.5)$ is an independent predictor of post-PCNL sepsis $(\mathrm{RR}=5.068, \mathrm{P}=0.033)$. A low $A G R$ is mainly used as a predictor of cancer progression and cancer-related mortality ${ }^{15}$. AGR mainly reflects the changes in plasma albumin and globulin levels. Among them, albumin is used to reflect the nutritional status and systemic inflammatory response; a lower level of albumin leads to the insufficient synthesis of immunoglobulin, which weakens the immune system ${ }^{16}$. Yang et al. reported that a low-normal level of serum albumin before surgery predicts post-PCNL fever ${ }^{17}$. Xu et al. demonstrated that the preoperative high- 
158

159

160

161

162

163

164

165

166

167

168

169

170

171

172

173

174

175

176

177

178

179

180

181

182

183

184

185

186

187

188

189

190

191

192

193

194

195

196

197

sensitive C-reactive protein/albumin (hs-CRP/Alb) ratio is independently predictive for the development of systemic inflammatory response syndrome after PCNL ${ }^{18}$. Usually, globulin plays an important role in immunity and inflammation. In the early phase of infection, the immunoglobulin level elevated rapidly ${ }^{19}$, which may account for the results in our study. A low AGR may indicate a susceptible state to infection. PCNL surgery brings about a stress response and backflow of pathogens and toxins, which accelerates the spread of infection and further leads to the occurrence of sepsis. Unlike other studies that only focus on local factors ${ }^{20,21}$, our study found that AGR, a systemic factor, maybe one of the important predictive factors of sepsis after PCNL.

Another systemic factor noted in our study to be closely related to sepsis after PCNL is preoperative leukocytosis $(\mathrm{RR}=3.706, \mathrm{P}=0.006)$. Mahmood et al. reported that preoperative leukocytosis is associated with adverse postoperative outcomes after cardiac surgery and is an independent predictor of infection-related postoperative complications ${ }^{22}$. Sen et al. found that the preoperative neutrophil-lymphocyte ratio may be a promising additive predictor of bacteremia and postoperative sepsis in patients who undergo PCNL for renal stones ${ }^{23}$. However, Bozkurt et al. presented a different view on the relationship between postoperative leukocytosis and sepsis. Their study showed that postoperative leukocytosis is common after PCNL and represents a normal physiologic response to surgery ${ }^{24}$. Preoperative leukocytosis may indicate the existence of potential infection in the body. The stress reaction and trauma caused by PCNL surgery may lead to the aggravation of infection, and thus, resulting in sepsis.

As a usual local urological condition, positive urine culture is considered as a critical factor related to infectious complications after a PCNL operation ${ }^{17,25}$. It is in conformity with our result that the risk of sepsis was found to be increased by more than three times in patients with positive urine culture. PCNL may cause vascular injury and high intrarenal pressure. For patients with a positive urine culture, this operation can promote the invasion of blood circulation by local pathogens and toxins and lead to the occurrence of sepsis. In our study, bladder urine was used for the urine culture examination. Emerging studies concluded that stone culture ${ }^{26,27}$ and pelvic urine culture ${ }^{27}$ were better predictors of urosepsis than mid-stream bladder urine culture. However, sample from kidney and stone cannot be obtained until surgery starts, which limits their early predictive and preventive value. Mid-stream bladder urine culture is still the optimal predictor that can be early acquired.

In our study, female sex was also found to be an independent predictor for sepsis after PCNL $(\mathrm{RR}=2.529, \mathrm{P}=0.024)$. Similarly, many studies have reported that female sex was significantly associated with post-URS or post - PCNL SIRS 18,25,28. Martov et al. from the CROES URS Global Study showed that female sex was a significant risk factor of postoperative urinary tract infection or fever in patients with a negative baseline urine culture ${ }^{29}$. Female is more susceptible to urinary tract infection than male, which may be one of the reasons why female sex is prone to sepsis after $\mathrm{PCNL}^{30}$. Additionally, sex-related gene polymorphisms ${ }^{31}$ and the effects of sex hormones ${ }^{32}$ may be other potential pathomechanisms to account for the susceptibility to sepsis after PCNL of the female sex. 
198 Our study had certain limitations. First, this study was a retrospective study performed at a single

199

200

201

202

203

204

205

206

207

208

209

210

211

212

213

214

\section{Acknowledgments}

216 None

217

218

219

220

221

222

223

224

225

226

227

228

229

230

231

232

233

234

235

236

237

238

239

240

241

\section{References} 2013;10(10):598-605. 2016;30(9):982-986. center, which may have led to potential selection bias. Second, only patients with proximal ureteral stone were enrolled considering homogeneous stone characteristics. Further research will investigate various kidney stone to verify the risk factors of sepsis. Third, although the model established in this study revealed favorable fitting, discrimination, and clinical usefulness, external validation is lacking, which is expected to be testified in independent cohorts. It is the first study to reveal the predictive value of the preoperative AGR for post - PCNL sepsis. However, the specific underlying mechanism remains unclear. A prospective study is planning to uncover the septic risk with more precise variables.

\section{Conclusions}

A new nomogram is developed to predict sepsis in PCNL for an upper ureteric single stone if the patient has these characters: female sex, lower AGR, positive urine culture, and leukocytosis.

1. Zeng G, Mai Z, Xia S, et al. Prevalence of kidney stones in China: an ultrasonography based cross-sectional study. BJU Int. 2017;120(1):109-116.

2. Scales CD, Smith AC, Hanley JM, Saigal CS. Prevalence of kidney stones in the United States. European urology. 2012;62(1):160-165.

3. Heinze A, Gozen AS, Rassweiler J. Tract sizes in percutaneous nephrolithotomy: does miniaturization improve outcome? Curr Opin Urol. 2019 Mar;29(2):118-123.

4. De S, Autorino R, Kim FJ, et al. Percutaneous nephrolithotomy versus retrograde intrarenal surgery: a systematic review and meta-analysis. European urology. 2015;67(1):125-137.

5. Chung DY, Kang DH, Cho KS, et al. Comparison of stone-free rates following shock wave lithotripsy, percutaneous nephrolithotomy, and retrograde intrarenal surgery for treatment of renal stones: A systematic review and network meta-analysis. PLoS One. 2019 Feb 21;14(2):e0211316.

6. Kreydin EI, Eisner BH. Risk factors for sepsis after percutaneous renal stone surgery. Nat Rev Urol.

7. Xu CG, Guo YL. Diagnostic and Prognostic Values of BMPER in Patients with Urosepsis following Ureteroscopic Lithotripsy. Biomed Res Int. 2019 January 20;2019:8078139.

8. Rivera M, Viers B, Cockerill P, Agarwal D, Mehta R, Krambeck A. Pre- and Postoperative Predictors of Infection-Related Complications in Patients Undergoing Percutaneous Nephrolithotomy. J Endourol.

9. Levy MM, Fink MP, Marshall JC, et al. 2001 SCCM/ESICM/ACCP/ATS/SIS International Sepsis Definitions Conference. Crit Care Med. 2003;31(4):1250-1256.

10. Steyerberg EW, Vergouwe Y. Towards better clinical prediction models: seven steps for development and an ABCD for validation. European heart journal. 2014;35(29):1925-1931. 
242

243

244

245

246

247

248

249

250

251

252

253

254

255

256

257

258

259

260

261

262

263

264

265

266

267

268

269

270

271

272

273

274

275

276

277

278

279

280

281

282

283

284

285

286

287

288

289

290

291

292

293

294
11. Wiesenthal JD1, Ghiculete D, D'A Honey RJ, Pace KT. A comparison of treatment modalities for renal calculi between 100 and $300 \mathrm{~mm}$ : are shockwave lithotripsy, ureteroscopy, and percutaneous nephrolithotomy equivalent? J Endourol. 2011;25(3):481-5.

12. De S, Autorino R, Kim FJ, et al. Percutaneous nephrolithotomy versus retrograde intrarenal surgery: a systematic review and meta-analysis. European urology. 2015;67(1):125-137.

13. Rivera M, Viers B, Cockerill P, et al. Pre- and Postoperative Predictors of Infection-Related Complications in Patients Undergoing Percutaneous Nephrolithotomy. J Endourol. 2016 Sep;30(9):982-6.

14. Shoshany O, Margel D, Finz C, et al. Percutaneous nephrolithotomy for infection stones: what is the risk for postoperative sepsis? A retrospective cohort study. Urolithiasis. 2015 Jun;43(3):237-42.

15. Lv GY, An L, Sun XD, Hu YL, Sun DW. Pretreatment albumin to globulin ratio can serve as a prognostic marker in human cancers: a meta-analysis. Clinica chimica acta; international journal of clinical chemistry. 2018;476:81-91.

16. McMillan DC, Watson WS, O'Gorman P, Preston T, Scott HR, McArdle CS. Albumin concentrations are primarily determined by the body cell mass and the systemic inflammatory response in cancer patients with weight loss. Nutrition and cancer. 2001;39(2):210-213.

17. Yang T, Liu S, Hu J, Wang L, Jiang H. The Evaluation of Risk Factors for Postoperative Infectious Complications after Percutaneous Nephrolithotomy. Biomed Res Int. 2017;2017:4832051.

18. $\mathrm{Xu} \mathrm{H,} \mathrm{Hu} \mathrm{L,} \mathrm{Wei} \mathrm{X,} \mathrm{et} \mathrm{al.} \mathrm{The} \mathrm{Predictive} \mathrm{Value} \mathrm{of} \mathrm{Preoperative} \mathrm{High-Sensitive} \mathrm{C-Reactive} \mathrm{Protein/Albumin}$ Ratio in Systemic Inflammatory Response Syndrome After Percutaneous Nephrolithotomy. J Endourol. 2019;33(1):1-8.

19. Nevo A, Mano R, Schreter E, Lifshitz DA. Clinical Implications of Stent Culture in Patients with Indwelling Ureteral Stents Prior to Ureteroscopy. The Journal of urology. 2017;198(1):116-121.

20. Rashid AO, Fakhulddin SS. Risk factors for fever and sepsis after percutaneous nephrolithotomy. Asian $J$ Urol. 2016 Apr;3(2):82-87.

21. Sharma K, Sankhwar SN, Goel A, et al. Factors predicting infectious complications following percutaneous nephrolithotomy. Urol Ann. 2016 Oct-Dec;8(4):434-438.

22. Mahmood E, Knio ZO, Mahmood F, et al. Preoperative asymptomatic leukocytosis and postoperative outcome in cardiac surgery patients. PloS one. 2017;12(9):e0182118.

23. Sen V, Bozkurt IH, Aydogdu O, et al. Significance of preoperative neutrophil-lymphocyte count ratio on predicting postoperative sepsis after percutaneous nephrolithotomy. The Kaohsiung journal of medical sciences. 2016;32(10):507-513.

24. Bozkurt IH, Aydogdu O, Yonguc T, et al. Predictive Value of Leukocytosis for Infectious Complications After Percutaneous Nephrolithotomy. Urology. 2015;86(1):25-29.

25. Uchida Y, Takazawa R, Kitayama S, Tsujii T. Predictive risk factors for systemic inflammatory response syndrome following ureteroscopic laser lithotripsy. Urolithiasis. 2018;46(4):375-381.

26. Eswara JR, Shariftabrizi A, Sacco D. Positive stone culture is associated with a higher rate of sepsis after endourological procedures. Urolithiasis. 2013 Oct;41(5):411-4.

27. Mariappan P, Smith G, Bariol SV, et al. Stone and pelvic urine culture and sensitivity are better than bladder urine as predictors of urosepsis following percutaneous nephrolithotomy: a prospective clinical study. J Urol. 2005 May;173(5):1610-4.28. Liu C, Zhang X, Liu Y, Wang P. Prevention and treatment of septic shock following mini-percutaneous nephrolithotomy: a single-center retrospective study of 834 cases. World J Urol. 2013;31(6):1593-1597.

29. Martov A, Gravas S, Etemadian M, et al. Postoperative infection rates in patients with a negative baseline urine culture undergoing ureteroscopic stone removal: a matched case-control analysis on antibiotic prophylaxis from the CROES URS global study. J Endourol. 2015;29(2):171-180.

30. Zhu C, Liu H, Wang Y, et al. Prevalence, incidence, and risk factors of urinary tract infection among immobile inpatients in China: a prospective, multi-centre study. The Journal of hospital infection. 2019.

31. Hubacek JA, Stuber F, Frohlich D, et al. Gene variants of the bactericidal/permeability increasing protein and lipopolysaccharide binding protein in sepsis patients: gender-specific genetic predisposition to sepsis. Crit Care Med. 2001;29(3):557-561.

32. Federman DD. The biology of human sex differences. The New England journal of medicine. 2006;354(14):1507-1514. 


\section{Table $\mathbf{1}$ (on next page)}

Table 1: Patients characteristics and univariable analysis of risk factors for postoperative sepsis after percutaneous nephrolithotomy. 
1 Table 1: Patients characteristics and univariable analysis of risk factors for post-operative sepsis after percutaneous

2 nephrolithotomy.

\begin{tabular}{|c|c|c|c|}
\hline Variable & Sepsis $(n=35)$ & Non-sepsis $(n=710)$ & $P$ value \\
\hline Age (yr), mean (SD) & $49.7(10.8)$ & $50.7(12.1)$ & 0.625 \\
\hline BMI $\left(\mathrm{kg} / \mathrm{m}^{2}\right)$, mean $(\mathrm{SD})$ & $23.8(3.7)$ & $23.9(3.0)$ & 0.863 \\
\hline Operation time (min), mean (SD) & $107.6(32.8)$ & $101.6(37.2)$ & 0.349 \\
\hline \multicolumn{4}{|l|}{ Sex, n (\%) } \\
\hline Male & $11(2.3)$ & $462(97.7)$ & \multirow[t]{2}{*}{$<0.001$} \\
\hline Female & $24(8.8)$ & $248(91.2)$ & \\
\hline \multicolumn{4}{|l|}{ Stone height, $\mathrm{n}(\%)$} \\
\hline$<20 \mathrm{~mm}$ & $29(4.6)$ & $601(95.4)$ & \multirow[t]{2}{*}{0.775} \\
\hline$\geq 20 \mathrm{~mm}$ & $6(5.2)$ & $109(94.8)$ & \\
\hline \multicolumn{4}{|l|}{ Stone laterality, n (\%) } \\
\hline Left & $18(4.6)$ & $372(95.4)$ & \multirow[t]{2}{*}{0.911} \\
\hline Right & $17(4.8)$ & $338(95.2)$ & \\
\hline \multicolumn{4}{|l|}{ Indwelling stent, $\mathrm{n}(\%)$} \\
\hline Yes & $3(6.0)$ & $47(94.0)$ & \multirow[t]{2}{*}{0.917} \\
\hline No & $32(4.6)$ & $663(95.4)$ & \\
\hline \multicolumn{4}{|l|}{ Hydronephrosis, n (\%) } \\
\hline Yes & $4(2.9)$ & 134(97.1) & \multirow[t]{2}{*}{0.268} \\
\hline No & $31(5.1)$ & $576(94.9)$ & \\
\hline \multicolumn{4}{|l|}{ Hypertension, n (\%) } \\
\hline Yes & $7(3.9)$ & $173(96.1)$ & \multirow[t]{2}{*}{0.556} \\
\hline No & $28(5.0)$ & $537(95.0)$ & \\
\hline \multicolumn{4}{|l|}{ Coronary heart disease, $\mathrm{n}(\%)$} \\
\hline Yes & $1(7.1)$ & 13(92.9) & \multirow[t]{2}{*}{1.000} \\
\hline No & $34(4.7)$ & $697(95.3)$ & \\
\hline \multicolumn{4}{|l|}{ Diabetes, n (\%) } \\
\hline Yes & $3(4.8)$ & $60(95.2)$ & \multirow[t]{2}{*}{1.000} \\
\hline No & $32(4.7)$ & $650(95.3)$ & \\
\hline \multicolumn{4}{|l|}{ Serum cholesterol, n (\%) } \\
\hline$<5.17 \mathrm{mmol} / \mathrm{L}$ & $34(5.2)$ & $617(94.8)$ & \multirow[t]{2}{*}{0.128} \\
\hline$\geq 5.17 \mathrm{mmol} / \mathrm{L}$ & $1(1.1)$ & 93(98.9) & \\
\hline \multicolumn{4}{|l|}{ Serum creatinine, $\mathrm{n}(\%)$} \\
\hline normal & $31(4.9)$ & $602(95.1)$ & \multirow[t]{2}{*}{0.541} \\
\hline abnormal & $4(3.6)$ & 108(96.4) & \\
\hline \multicolumn{4}{|l|}{ Albumin, n (\%) } \\
\hline$<35 \mathrm{~g} / \mathrm{L}$ & $10(11.2)$ & $79(88.8)$ & \multirow[t]{2}{*}{0.005} \\
\hline$\geq 35 \mathrm{~g} / \mathrm{L}$ & $25(3.8)$ & $631(96.2)$ & \\
\hline Globulin, n (\%) & & & 0.001 \\
\hline
\end{tabular}




\begin{tabular}{|c|c|c|c|}
\hline$<30 \mathrm{~g} / \mathrm{L}$ & $12(2.6)$ & $450(97.4)$ & \\
\hline$\geq 30 \mathrm{~g} / \mathrm{L}$ & $23(8.1)$ & $260(91.9)$ & \\
\hline \multicolumn{4}{|l|}{ AGR, n (\%) } \\
\hline$<1.5$ & $33(6.7)$ & $457(93.3)$ & \multirow[t]{2}{*}{$<0.001$} \\
\hline$\geq 1.5$ & $2(0.8)$ & 253(99.2) & \\
\hline \multicolumn{4}{|c|}{ Pre-operative fever, n (\%) } \\
\hline Yes & $9(17.0)$ & $44(83.0)$ & \multirow[t]{2}{*}{$<0.001$} \\
\hline No & $26(3.8)$ & $666(96.2)$ & \\
\hline \multicolumn{4}{|l|}{ WBC, n (\%) } \\
\hline$<10,000$ cells $/ \mu \mathrm{L}$ & $25(3.7)$ & $659(96.3)$ & \multirow[t]{2}{*}{$<0.001$} \\
\hline$\geq 10,000$ cells $/ \mu \mathrm{L}$ & $10(16.4)$ & $51(83.6)$ & \\
\hline \multicolumn{4}{|l|}{ Urine culture, n (\%) } \\
\hline Positive & 13(14.9) & $74(85.1)$ & \multirow[t]{2}{*}{$<0.001$} \\
\hline Negative & $22(3.3)$ & $636(96.7)$ & \\
\hline \multicolumn{4}{|l|}{ Urine WBC, n (\%) } \\
\hline$<50$ cells $/ \mu \mathrm{L}$ & $7(1.9)$ & $353(98.1)$ & \multirow[t]{2}{*}{0.001} \\
\hline$\geq 50$ cells $/ \mu \mathrm{L}$ & $28(7.3)$ & $357(92.7)$ & \\
\hline \multicolumn{4}{|l|}{ Urine nitrite, n (\%) } \\
\hline Positive & $8(17.8)$ & $37(82.2)$ & \multirow[t]{2}{*}{$<0.001$} \\
\hline Negative & $27(3.9)$ & $673(96.1)$ & \\
\hline ASA score, n (\%) & & & 0.591 \\
\hline 1 & $18(5.9)$ & $286(94.1)$ & \\
\hline 2 & $17(4.0)$ & 408(96.0) & \\
\hline 3 & $0(0.0)$ & $15(100.0)$ & \\
\hline 4 & $0(0.0)$ & $1(100.0)$ & \\
\hline
\end{tabular}

3 Abbreviations: BMI, body mass index; SD, standard deviation; AGR, albumin globulin ratio; WBC, white blood cell.

4 
Table 2 (on next page)

Table 2: Multivariable logistic regression analysis of predictors of sepsis after percutaneous nephrolithotomy. 
1 Table 2: Multivariable logistic regression analysis of predictors of sepsis after percutaneous nephrolithotomy.

\begin{tabular}{lccccc}
\hline Variables & $\mathrm{B}$ & $\mathrm{SE}$ & OR & $95 \% \mathrm{CI}$ & $P$ value \\
\hline Age $(\mathrm{yr})$ & -0.021 & 0.018 & 0.979 & $0.946-1.014$ & 0.240 \\
BMI $\left(\mathrm{kg} / \mathrm{m}^{2}\right)$ & 0.021 & 0.059 & 1.021 & $0.909-1.148$ & 0.720 \\
Operation time $(\mathrm{h})$ & 0.273 & 0.297 & 1.314 & $0.734-2.351$ & 0.358 \\
Stone height $\geq 20 \mathrm{~mm}$ & 0.034 & 0.502 & 1.035 & $0.387-2.768$ & 0.945 \\
Pre-operative fever & 0.828 & 0.476 & 2.288 & $0.900-5.820$ & 0.082 \\
Indwelling stent & -0.728 & 0.684 & 0.483 & $0.127-1.844$ & 0.287 \\
Diabetes & -0.010 & 0.700 & 0.990 & $0.251-3.906$ & 0.989 \\
Hydronephrosis & 0.214 & 0.572 & 1.238 & $0.404-3.797$ & 0.709 \\
AGR $<1.5$ & 1.623 & 0.763 & 5.068 & $1.135-22.624$ & 0.033 \\
Positive urine culture & 1.176 & 0.523 & 3.243 & $1.162-9.047$ & 0.025 \\
leukocytosis & 1.310 & 0.481 & 3.706 & $1.444-9.512$ & 0.006 \\
Positive urine nitrite & 0.052 & 0.581 & 1.053 & $0.337-3.287$ & 0.929 \\
Female & 0.928 & 0.412 & 2.529 & $1.127-5.672$ & 0.024 \\
Urine WBC $\geq 50$ cells $/ \mu$ & 0.706 & 0.481 & 2.026 & $0.789-5.197$ & 0.142 \\
\hline
\end{tabular}

2 Abbreviations: AGR, albumin globulin ratio; WBC, white blood cell; B, regression coefficient; SE, standard error;

3 OR, Odds Risk; CI, confidence interval. 
Figure 1

Categorized albumin globulin ratio (AGR) and corresponding sepsis rate (***P <0.001).

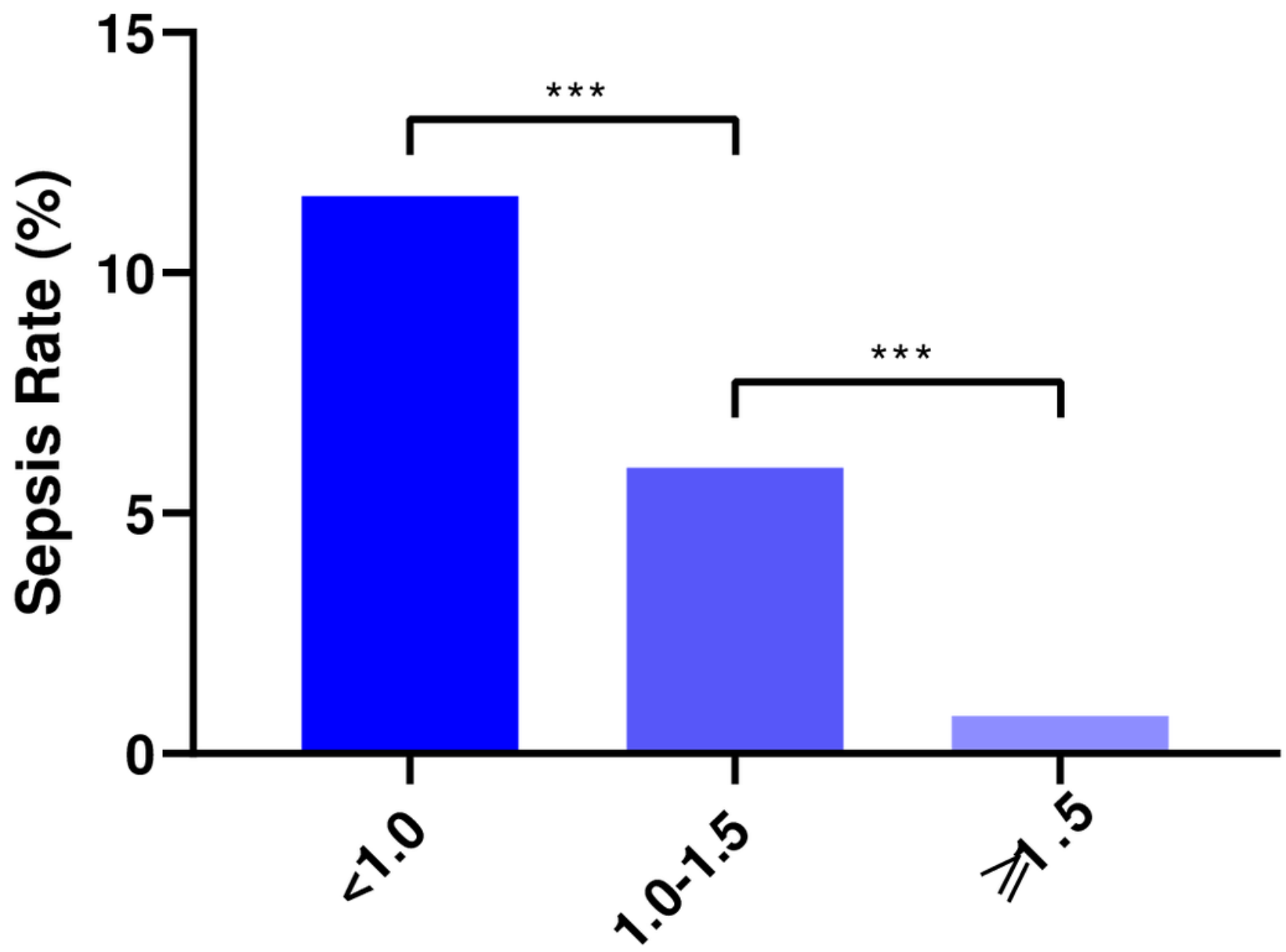

AGR 
Figure 2

Nomogram for patients predicting post-operative sepsis.

WBC, white blood cell; AGR, albumin globulin ratio. Sex, urine culture, WBC and AGR are marked as "points". Total points by adding the three points can predict sepsis rate.

Points

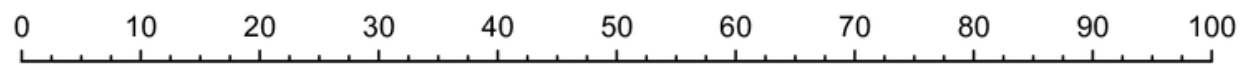

Sex

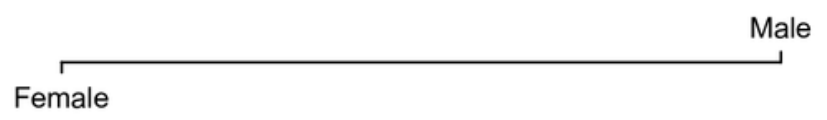

UrineCulture

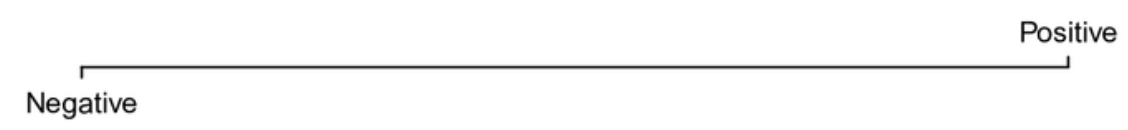

WBC

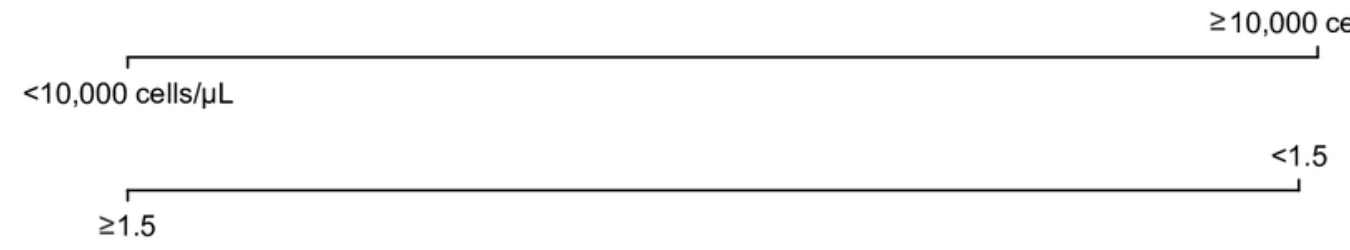

Total Points

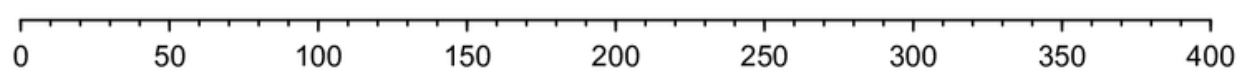

Sepsis rate

$\begin{array}{lllllll}0.05 & 0.1 & 0.2 & 0.3 & 0.4 & 0.5 & 0.6\end{array}$


Figure 3

Evaluation of the predictive performance.

(A) Calibration curve. Hosmer-Lemeshow test with insignificant $P$ value indicates good fitting of model. (B) Receiver operating characteristic (ROC) curve. The area under curve (AUC) for the model is 0.807 , which showed a favorable ability of discrimination.

A. Calibration curve

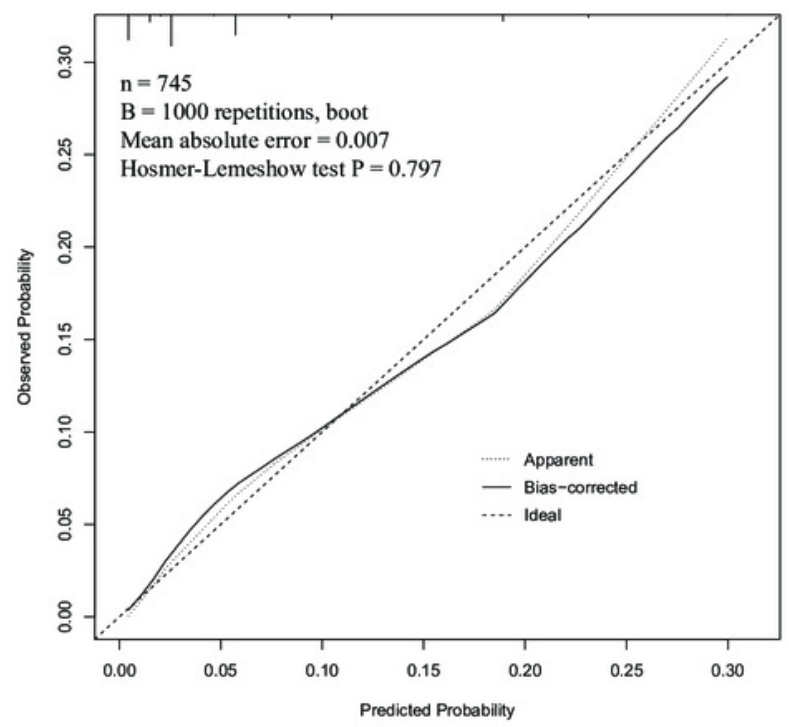

B. ROC curve

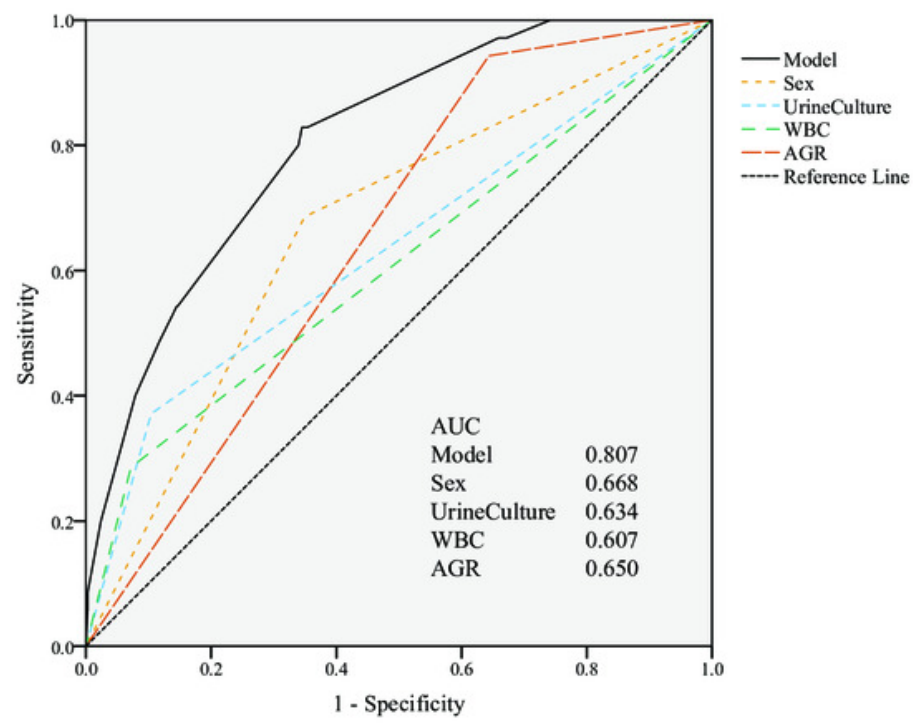


Figure 4

Decision curve analysis (DCA).

When risk threshold is around $10-90 \%$, the net benefit of application of the model on taking measures is greater than "treat-all-patient" or "treat-none" scheme. In addition, utilization of sex, urine culture, white blood cell (WBC) and albumin globulin ratio (AGR) alone is inferior than the model.

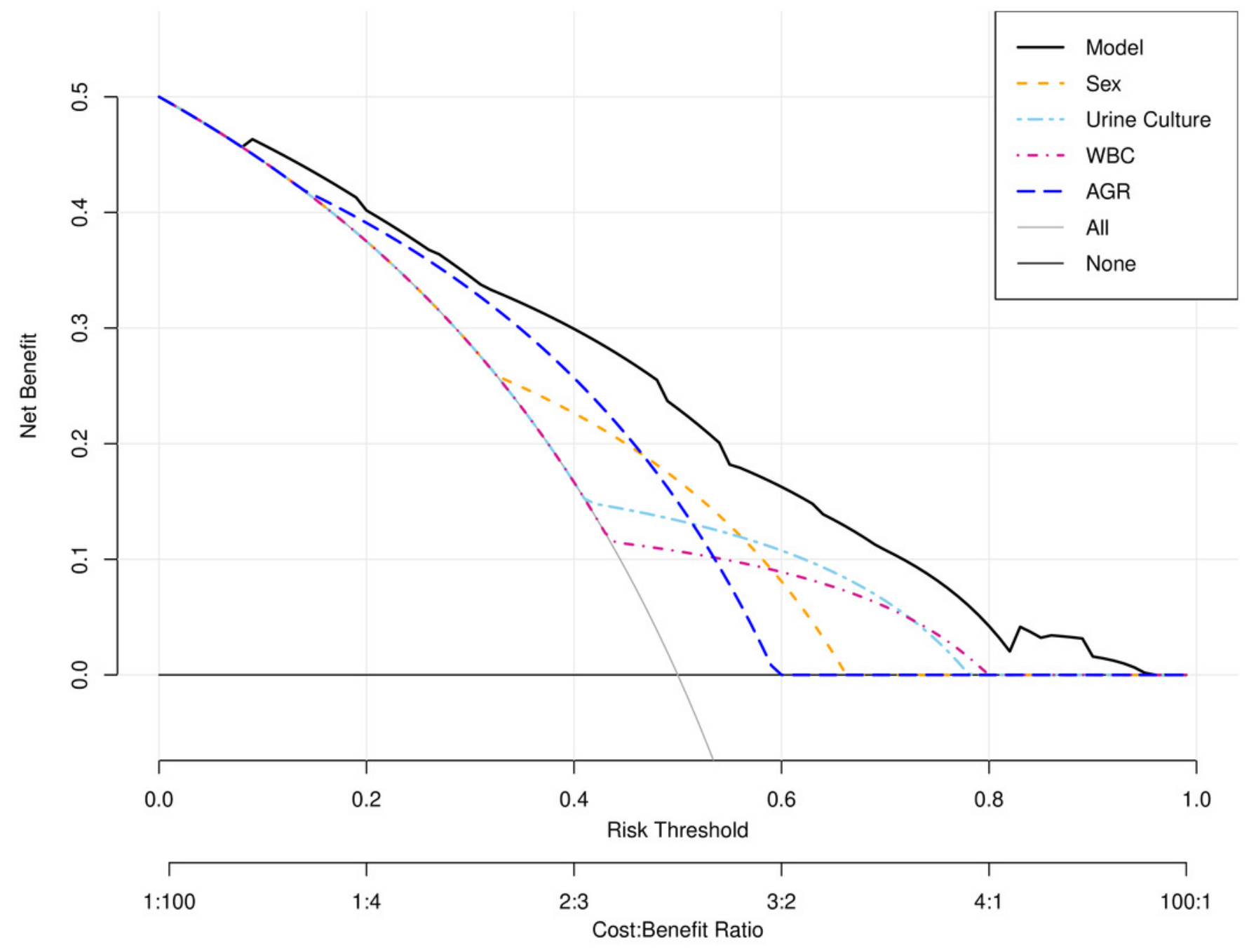

